\title{
Anti-CD44 mAb remodels biological behaviors of spheroid cells with stemness from human ovarian cancer cell line SKOV-3
}

\author{
GU Chao ${ }^{1}$, DU YongRui ${ }^{2}$, GAO Yan ${ }^{2}$, YAO Zhi $^{1}$, GU Xin ${ }^{1}$, ZHANG QiuYue $^{1}$, XU JingJing $^{1} \&$ \\ DENG WeiMin ${ }^{1 *}$
}

${ }^{1}$ Tianjin Key Laboratory of Cellular and Molecular Immunology, Tianjin Medical University, Tianjin 300070, China;
${ }^{2}$ Department of Gynecology, Tianjin Central Gynecology and Obstetrics Hospital, Tianjin 300100, China

Received December 9, 2011; accepted January 18, 2012

\begin{abstract}
There is accumulating evidence that cancer stem cells (CSCs) play an important role in tumor progression. Novel strategies targeting CSCs have been widely researched. In the present study, we explored whether such CSCs existed in human ovarian cancer (OVCA) cell line and whether anti-CD44 antibody had effects on such subpopulation. We isolated and identified spheroid cells from SKOV-3. Then we used A3D8, an anti-CD44 mAb to treat spheroid cells with so-called "stemness". Effects of A3D8 on spheroid cells' biological behaviors were examined. Our findings showed that there was a small subpopulation that had so-called "stemness" in SKOV-3 cell line. Against spheroid cells, A3D8 can (1) inhibit cell proliferation; (2) change cell cycle distribution and expression of p21, CDK2 and cyclinA; (3) enhance cisplatin (DDP)-induced apoptosis; (4) promote cell differentiation; (5) inhibit clone formation efficiency; (6) reduce invasive efficacy; (7) inhibit tumorigenicity. Thus, to sum up points which we have just showed, spheroid cells isolated from SKOV-3 can be used as an appropriate in vitro model for relevant study of human ovarian CSCs. And our results reasoned that anti-CD44 therapy may become a potential promising strategy for OVCA treatment.
\end{abstract}

cancer stem cell (CSC), targeting therapy, CD44, antibody, ovarian cancer (OVCA)

Citation: $\quad$ Gu C, Du Y R, Gao Y, et al. Anti-CD44 mAb remodels biological behaviors of spheroid cells with stemness from human ovarian cancer cell line SKOV-3. Chin Sci Bull, 2012, 57: 1288-1297, doi: 10.1007/s11434-012-5075-5

Recently, there is accumulating evidence that cancer stem cells (CSCs) or cancer progenitor cells play an important role in cancer initiation, progression, metastasis and resistance to conventional therapies (including tumor surgical ablation, radiotherapy and chemotherapy, alone or in combination). Therefore it is necessary to explore new strategies targeting these tumor stem/progenitor cells to improve the efficacy of cancer treatment [1-7]. Currently, use of anti-CD44 mAb as a potential treatment strategy targeting leukemia stem cells (LSCs) as well as leukemia cells has been extensively studied. Many research groups reported that anti-CD44 mAb had multiple effects on LSCs as well as leukemia cells, such as promoting differentiation, inhibiting proliferation, inducing apoptosis in vitro and decrease potential of tumorigenicity [8-12]. However, whether an-

*Corresponding author (email: dengwm67@yahoo.com.cn)
ti-CD44 mAb has similar effects on CSC population in human OVCA which is the leading cause of death among gynecological neoplasms in the world was still unclear. Recent publications reported that there was a minor subpopulation with "stemness" in primary human ovarian tumor bulk [13] and ascites sample [14]. Moreover, this small subpopulation has been considered to be responsible for OVCA progression. But up to date, whether this subpopulation exists in well-established human OVCA cell line, and whether anti-CD44 mAb is effective on such subpopulation, are still unknown and need to be explored. Therefore, in our present study, we managed to isolate spheroid cells from SKOV-3, a widely-used and a well-established human OVCA cell line, and evaluated their stem cell-like biological behaviors. Subsequently, we used A3D8, one widely-used anti-CD44 $\mathrm{mAb}$ against all CD44 isoforms [15], to treat spheroid cells to evaluate therapeutic effects of anti-CD44 mAb on stem 
cell-like subpopulation.

\section{Materials and methods}

\subsection{Cell line and culture}

Human OVCA cell line SKOV-3 (ATCC) was cultured in RPMI1640 supplemented with $10 \%$ fetal bovine serum (FBS; Sigma, USA), penicillin, streptomycin and $L$-glutamine. The cell line was grown in $\log$ phase at $37^{\circ} \mathrm{C}$ in $5 \%$ $\mathrm{CO}_{2}$ atmosphere. SKOV-3 cells grew as adherent monolayer and then were regularly digested with $0.25 \%$ trypsin containing $0.02 \%$ EDTA to make single cell suspension for relevant assays.

\subsection{Spheroid cells isolation and culture}

Cell culture and passages were conducted following published protocols $[13,14]$. In brief, adherent SKOV-3 cells were gently trypsinized into single cell suspension and seeded in $\mathrm{T}_{25}$ culture flask at a density of $4 \times 10^{3}$ cells $/ \mathrm{cm}^{2}$ in serum-free DMEM/F12 culture medium supplemented with $5 \mu \mathrm{g} / \mathrm{mL}$ insulin (Sigma), $1 \mathrm{ng} / \mathrm{mL}$ hydrocortisone (Sigma), $20 \mathrm{ng} / \mathrm{mL}$ human recombinant epidermal growth factor (hrEGF; Invitrogen, USA), $10 \mathrm{ng} / \mathrm{mL}$ basic fibroblast growth factor (bFGF; Invitrogen), $5 \mathrm{ng} / \mathrm{mL}$ stem cell factor (SCF; Invitrogen), 2\% B27 and $0.4 \%$ bovine serum albumin (BSA; Sigma). After 5-7 d incubation, primary spheroids were observed. The primary spheroid cells were trypsinized and further cultured. The secondary spheroids were isolated for further expansion.

\subsection{Cell proliferation rate assay}

Freshly trypsinized single spheroid cells were seeded in 96-well culture plates in $100 \mu \mathrm{L}$ serum-free medium described above at a density of $1 \times 10^{4}$ cells/well. The cells were treated with different concentration of mAb A3D8 (Sigma) $(1,10,50 \mu \mathrm{g} / \mathrm{mL})$ for $48 \mathrm{~h}$. Three hours before the end of incubation, MTT was added into each well (final concentration of MTT is $5 \mathrm{mg} / \mathrm{mL}$ ). At the end of incubation, medium in each well was removed and deposit was dissolved in $150 \mu \mathrm{L}$ DMSO. The relative formazane concentration was measured by determination of absorbance at 573 nm (Well-plate Reader, Thermo). Mouse IgG1 (mIgG1) was used as isotype control.

\subsection{Immunocytochemistry assay}

Freshly trypsinized spheroid cells were seeded in 24-well plate containing glass coverslip in serum-free medium mentioned above $\left(1 \times 10^{5}\right.$ cells/mL per well), followed by treating with $10 \%$ serum, mIgG1 or A3D8 $(10 \mu \mathrm{g} / \mathrm{mL})$ for $7 \mathrm{~d}$ respectively. Morphology of cells was observed under microscope (Olympus, Japan) every day. The expression of
CK-7 and CA125 was assessed by regular immunocytochemistry assay respectively. The antibodies included mouse anti-human CA125 mAb (Santa Cruz), mouse antihuman CK-7 mAb (Santa Cruz) and horseradish peroxidase-labeled goat anti-mouse IgG secondary antibody (Santa Cruz). Diaminobezidin (DAB) Kit (Santa Cruz) was used as color indicator system. Quantification of cells stained with each antibody (brown color) was estimated as a percentage of brown cells in total cells in each microscopic field. Cells were counted in at least 5 microscopic fields per specimen.

\subsection{Analysis of ALDH enzymatic activity}

Analysis of aldehyde dehydrogenase (ALDH) enzymatic activity was performed using Aldefluor system (Miltenyi) following manufacturer's instructions. Briefly, cells were trypsinized and resuspended in phosphate buffer solution (PBS) supplemented with $2 \%$ FBS at a concentration of $1 \times 10^{6}$ cells $/ \mathrm{mL}$. One milliliter of above cell resuspension was mixed with $2 \mu \mathrm{L}$ Aldefluor substrate, then half of resuspended cells were immediately placed into a fresh vial with $5 \mu \mathrm{L}$ diethylaminobenzaldehyde (DEAB), a specific inhibitor of $\mathrm{ALDH}$ for negative control. Both tubes were incubated at $37^{\circ} \mathrm{C}$ for $30 \mathrm{~min}$. After incubation, cells were resuspended in the above fresh buffer and analyzed. Analysis was performed by using a flow cytometer (FACSCalibur, BD) equipped with a $488 \mathrm{~nm}$ argon ion laser for excitation and a $525 \mathrm{~nm}$ band pass filter. $\mathrm{ALDH}^{+}$cells are identified as cells with low side scatter and high green fluorescence.

\subsection{Apoptosis detection}

The percentage of apoptotic cells was detected by flow cytometry using PI/Annexin V-FITC Kit (Keygen, China) according to manufacturer's recommendations. Briefly, cells were harvested and washed twice with PBS containing $2 \%$ FBS, followed by staining with annexin V-fluorescein isothiocyanate (FITC) and propidium iodide (PI). Flow cytometry analysis was last performed. The compensation was modified using cells labeled with annexin V-FITC or with PI alone. Ten thousand of events were acquired for each sample. CellQuest Pro software (BD, USA) was used to acquire and analyze data.

\subsection{Cell cycle analysis}

Cells were fixed with $70 \%$ ethanol overnight at $-20^{\circ} \mathrm{C}$ and incubated with RNase $(50 \mu \mathrm{g} / \mathrm{mL})$ and PI $(2.5 \mu \mathrm{g} / \mathrm{mL})$ for $30 \mathrm{~min}$ at $4^{\circ} \mathrm{C}$ followed by flow cytometry. CellQuest Pro software was used to acquire data and results obtained were analyzed using ModFit LT Version 3.2 software to determine percentage of cells in G0/G1, S, G2/M phases of cell cycle [16]. 


\subsection{Cell extracts and Western blot analysis}

Proteins were extracted from spheroid cells and adherent SKOV-3 cells using Protein Extract Kit (Pierce). Total protein concentrations in each fraction were determined with BCA Protein Assay Kit (Pierce). Identical amounts of protein were separated on $12 \%$ SDS/PAGE $(50 \mu \mathrm{g} / \mathrm{lane})$ and electroblotted onto polyvinylidene fluoride membrane (Pall). The transfer was monitored by a prestained protein molecular weight marker (Invitrogen). After transfer, membranes were blocked with $5 \%$ skim milk in $50 \mathrm{mmol} / \mathrm{L}$ Tris- $\mathrm{HCl}$ (pH 7.4), $150 \mathrm{mmol} / \mathrm{L} \mathrm{NaCl}$, and $0.05 \%$ Tween-20 (TBS) for $1 \mathrm{~h}$ at room temperature. Immunoblotting was performed by incubation with a specific primary antibody overnight at $4^{\circ} \mathrm{C}$. Primary antibodies included mouse mAbs against human Nanog, Oct-4, ABCG2, p21, CDK2, cyclinA and bcl-2 (Santa Cruz). After washing three times for 30 min in TBS, membranes were secondly hybridized to peroxidase-labeled goat anti mouse antibody for detection with ECL chemoluminescence system (Santa Cruz).

\subsection{Analysis of mitochondrial transmembrane poten- tial $(\Delta \psi M)$}

Mitochondrial transmembrane potential was measured by flow cytometry using Rhodamine 123 (Rh123) Detection Kit (Keygen). Briefly, harvested cells were washed twice with PBS and incubated with $1 \mu \mathrm{g} / \mathrm{mL}$ Rh 123 at $37^{\circ} \mathrm{C}$ for 30 min. Cells were then washed twice with PBS, and Rh123 intensity was determined by flow cytometry. Cells with reduced fluorescence (less Rh123) were counted as having lost some of their mitochondrial transmembrane potential.

\subsection{Clone formation assay}

Freshly trypsinized spheroid cells were seeded in 96-well plate $\left(1 \times 10^{4}\right.$ cells $/ \mathrm{mL}, 100 \mu \mathrm{L} /$ well $)$, and then treated with $\mathrm{mIgG1}$ or A3D8 $(10 \mu \mathrm{g} / \mathrm{mL})$ for $48 \mathrm{~h}$ respectively. Each kind of above pretreated cells were trypsinized and recounted, and then soft-agar colony formation assay was performed according to what we described previously [17]. Briefly, cells were seeded in triplicate in 6-well plate with $1 \times 10^{4}$ cells/well and incubated in soft agar for $14 \mathrm{~d}$ at $37^{\circ} \mathrm{C}$ using a two layer agar system and clones were counted. The clone formation efficiency (CFE) was ratio of clone number to planted cell number.

\subsection{In vitro matrigel invasion assay}

Matrigel invasion assay was performed in 24-well invasion chamber system (Costar) of $6.5 \mathrm{~mm}$ diameter, with $8 \mu \mathrm{m}$ pore filters. Briefly, filter membrane was coated overnight at $4^{\circ} \mathrm{C}$ with fibronectin (FN, $\left.20 \mu \mathrm{g} / \mathrm{mL}, \mathrm{BD}\right)$ and then coated with matrigel $(200 \mu \mathrm{g} / \mathrm{mL}, 50 \mu \mathrm{L} / \mathrm{well}, \mathrm{BD})$ at $37^{\circ} \mathrm{C}$ for 1 h. A total of $1 \times 10^{5}$ spheroid cells were pretreated with
$\mathrm{mIgG1}$ or A3D8 $(10 \mu \mathrm{g} / \mathrm{mL})$ for $48 \mathrm{~h}$ respectively, and then were trypsinized and recounted. A total of $1 \times 10^{5}$ cells pretreated above in $0.1 \mathrm{~mL}$ assay medium (DMEM/F12 with $0.25 \%$ BSA) were added to upper compartment, and $0.6 \mathrm{~mL}$ DMEM/F12 medium supplemented with growth factors in the presence of 5\% FBS was added to lower compartment. After 48-h incubation, remaining tumor cells on upper surface of filters were removed by wiping with cotton swabs, and invading cells on lower surface were stained using regular HE staining. The cells number on lower surface of filters was counted under a microscope (Nikon, Japan). Data were obtained from three individual experiments in triplicate.

\subsection{Evaluation of spheroid cells' tumorigenicity in NOD/SCID mice}

Freshly trypsinized spheroid cells and adherent SKOV-3 cells were resuspended in serum-free culture described above. Cells were diluted to appropriate injection doses, and injected subcutaneously in NOD/SCID mice on the side of each flank. Briefly 12 NOD/SCID mice were randomly grouped into 6 groups, 2 mice per group. The spheroid cells $\left(1 \times 10^{2}, 1 \times 10^{3}\right.$ or $\left.1 \times 10^{4}\right)$, and SKOV- 3 adherent cells $\left(1 \times 10^{4}\right.$, $1 \times 10^{5}$ or $1 \times 10^{6}$ ) were respectively inoculated into NOD/ SCID mice as described above. Mice in each group were monitored. When tumors reached a maximum diameter of $15 \mathrm{~mm}$ or up to 4 months after cell inoculation without obvious tumor occurrence, the mice would be sacrificed and tumors were retrieved at the end of experiment for pathologic analysis.

\subsection{Animal test for effect of A3D8 on tumorigenicity}

Freshly trypsinized spheroid cells were seeded in 6-well plates in $1 \mathrm{~mL}$ of serum-free medium described above at a density of $1 \times 10^{5}$ cells $/ \mathrm{mL}$. After 24 -h regular incubation, cells were then treated with A3D8 at $10 \mu \mathrm{g} / \mathrm{mL}$ or $\mathrm{mIgG} 1$ at the same concentration as control. Twelve six-week-old NOD/SCID mice were randomly grouped into four groups, three mice per group. Each group of mice were injected subcutaneously on the flank with A3D8-pretreated spheroid cells $\left(1 \times 10^{4}\right.$ or $\left.1 \times 10^{5}\right)$ or $\mathrm{mIgG1}$-pretreated spheroid cells $\left(1 \times 10^{4}\right.$ or $\left.1 \times 10^{5}\right)$ suspended in PBS. The animals were observed daily. When tumors' diameter of control group mice reached $15 \mathrm{~mm}$, mice were sacrificed and tumors were retrieved at the end of experiment for pathologic analysis. All experiments involving use of animals were performed in accordance with Tianjin Medical University institutional animal welfare guidelines.

\subsection{Statistical methods}

Microsoft Office Excel 2007 and statistical software SPSS 16.0 were used in data processing and in analyzing signifi- 
cance between groups with paired $t$ test. $P<0.05$ was considered significant. The results were presented as mean \pm $\mathrm{SD}$ of three independent experiments.

\section{Results}

\subsection{The spheroid cells from SKOV-3 have so-called stemness}

To identify whether spheroid cells isolated from SKOV-3 had stemness, a batch of tests were performed. The data showed that spheroid cells exhibited the ability to unlimitedly divide in an anchorage-independent spheroid manner in serum-free medium supplemented with growth factors for at least 6 months without obvious phenotype change. This growth pattern is regarded as a growth manner of cancer stem/ progenitor cells under stem cell-selective conditions $[13,14]$. After passages, spheroid cells persisted as larger, symmetric prototypical spheroids. Typical spheroids contained 50-100 viable cells (Figure 1(a)). Considering cell cycle event characteristics of stem/progenitor cells, we also detected cell-cycle events of spheroid cells (SKOV-3 cells as control) by regular PI staining by flow cytometry. The data showed that about $85.23 \pm 2.36 \%$ spheroids and 55.03 \pm
4.07\% SKOV-3 cells presented in G0/G1 phase (Figure 1(b)). This result is consistent with the studies by Matsui et al. [18] and Takubo et al. [19] on cell cycle event of stem/ progenitor cells. As to the induction of spheroid cells' differentiation, according to Zhang et al. [13], spheroids were cultured under differentiating conditions (e.g., addition of $10 \%$ FBS after growth factors' withdrawal) for $7 \mathrm{~d}$, floating spheroids could adhere to culture plate, acquire an epithelial morphology and express epithelial markers CK-7 and ovarian CA125. And percentage of CK-7 positive and CA125 positive cells in total cell event were $50 \pm 6 \%$ and $45 \pm 5 \%$ respectively (Figure 1(c)). In addition, genes expression of Oct-4 and Nanog are regarded as marker of embryonic stem cells and stem cell expansion [14,20], so expression of Nanog and Oct-4 in spheroid cells were examined by Western blot assay. The result showed that, compared with adherent SKOV-3 cells, spheroid cells showed significantly stronger signal. Moreover, expression of $A B C G 2$, encoding a membrane efflux transporter and expressed in hematopoietic stem cells and associated with chemotherapy resistance [20], was also assessed by the same method. Results showed that spheroid cells expressed stem cell-relevant marker (Figure 1(d)), providing further evidence for their undifferentiated phenotype. Recently, there is accumulating evidence

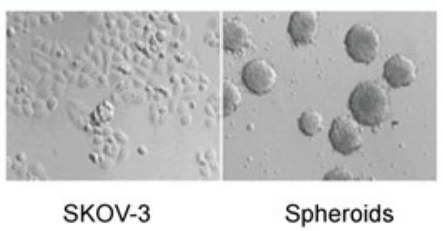

(a)

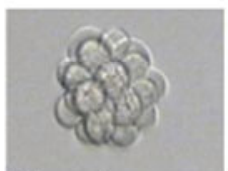

Secondary spheroids

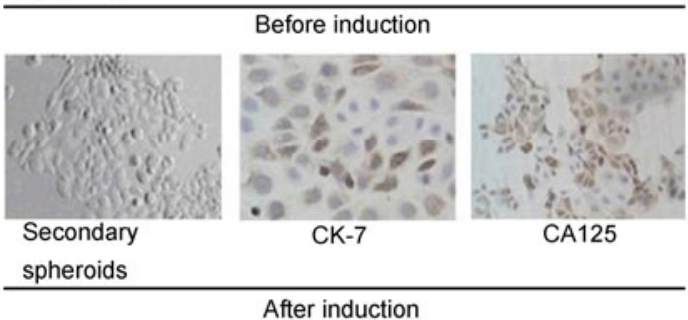

(c)

SKOV-3
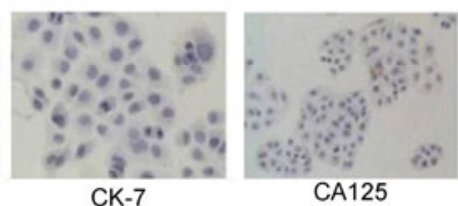

A125

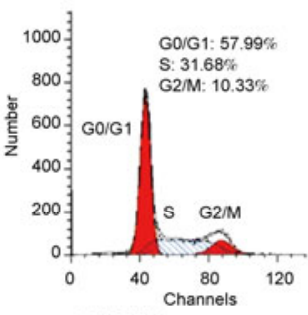

Nanog

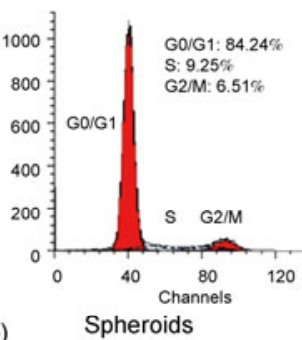

(b)

Spheroids

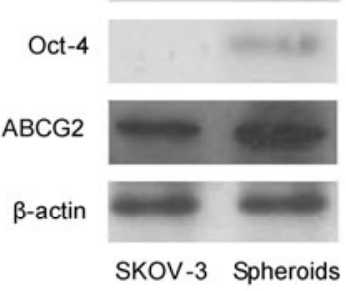

(d)

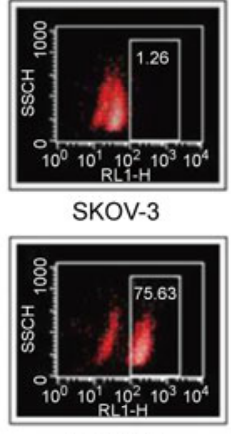

Spheroids

(e)

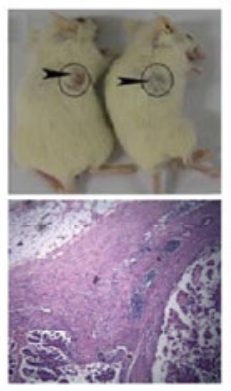

(f)

Figure 1 (Color online) Under stem cell selective-condition, spheroid cells from SKOV-3 displayed stem cell characteristics. (a) Morphology result of adherent SKOV-3 cells cultured in standard 10\% FCS RPMI 1640 medium and typical spheroids in serum-free selective medium supplemented with growth factors after passages. (b) Cell cycle event of spheroids and SKOV-3 cells by regular PI staining using flow cytometry. (c) Morphology, expression of CK-7 and CA125 in spheroid cells by immunocytochemistry versus differentiated cells cultured under differentiating conditions. (d) Expression of Nanog, Oct-4, and ABCG2 in spheroid cells versus adherent SKOV-3 cells by Western blot analysis. (e) Flow cytometry analysis of ALDH ${ }^{+}$cells in spheroid cells. (f) Xenograft tumor formed after injection of spheroid cells. Injection of $1 \times 10^{4}$ spheroid cells per mouse subcutaneously on the back of NOD/SCID mice generated tumors with $2 / 2$ efficiency. HE staining sections of primary tumor reproduced by $1 \times 10^{4}$ spheroid cells. 
that ALDH, an enzyme involved in stem cell self-protection, is regarded as another important property of stem cells [21]. Therefore we further detected ALDH activity of spheroid cells by flow cytometry. The data exhibited that about $75 \%$ of spheroid cells was $\mathrm{ALDH}^{+}$cells and had a high level of ALDH activity (Figure 1(e)). The last but not least of all, tumorigenicity of spheroid cells in NOD/SCID mice was also assessed. Results showed that as few as $1 \times 10^{4}$ spheroid cells injected into NOD/SCID had the ability to reproduce tumor exhibiting similar phenotype to that reproduced by $1 \times 10^{6}$ adherent SKOV-3 cells. This result indicated that, compared with SKOV-3 cells, exponentially smaller number of spheroid cells could reproduce tumor (Figure 1(f) and Table 1).

\subsection{A3D8 inhibited spheroid cell proliferation rate}

To evaluate whether A3D8 has effects on proliferation of spheroid cells, MTT assay was performed. The data showed that A3D8 significantly decreased proliferation rate of spheroid cells in a dose-dependent manner $(P<0.05)$ (Figure 2).

\subsection{A3D8 decreased fraction of spheroid cells in G0/G1 phase and regulated expression of p21, CDK2 and cy- clinA}

To determine whether A3D8 can affect cell cycle distribution of spheroid cells, we treated spheroid cells with A3D8 at $10 \mu \mathrm{g} / \mathrm{mL}$ for $48 \mathrm{~h}$, and cell cycle was then analyzed. Plot data showed that $\mathrm{G} 0 / \mathrm{G} 1$ percentages of $\mathrm{mIgG} 1$ or A3D8-treated spheroid cells were $86.02 \pm 2.95 \%$ and $59.70 \pm 3.25 \%$, respectively $(P<0.05)$; while those in $\mathrm{S}$ phase were $7.82 \pm 0.90 \%$ and $28.97 \pm 3.65 \%$, respectively $(P<0.05)$ (Figure 3(a-c)). Western blot showed that A3D8 upregulated expression of p21 while downregulated CDK2 and cyclinA levels $(P<0.05)$ (Figure 3(d) and (e)).

\subsection{A3D8 enhanced DDP-induced apoptosis of sphe- roid cells}

In the present study, we treated spheroid cells with 10 $\mu \mathrm{g} / \mathrm{mL} \mathrm{mIgG} 1,10 \mu \mathrm{g} / \mathrm{mL}$ A3D8 at $2.5 \mu \mathrm{g} / \mathrm{mL}$ DDP, or 10

Table 1 Tumorigenicity of spheroid cells and SKOV-3 cells in NOD/ SCID mice

\begin{tabular}{ccc}
\hline Groups & Cell dose & Tumor formation $^{\text {a) }}$ \\
\hline \multirow{3}{*}{ Spheroid cells } & $1 \times 10^{2}$ & $0 / 2$ \\
& $1 \times 10^{3}$ & $1 / 2$ \\
& $1 \times 10^{4}$ & $2 / 2$ \\
\hline \multirow{2}{*}{ SKOV-3 cells } & $1 \times 10^{4}$ & $0 / 2$ \\
& $1 \times 10^{5}$ & $0 / 2$ \\
& $1 \times 10^{6}$ & $0 / 2$ \\
\hline
\end{tabular}

a) Two mice per group. $\mu \mathrm{g} / \mathrm{mL}$ A3D8 plus $2.5 \mu \mathrm{g} / \mathrm{mL}$ DDP for $24 \mathrm{~h}$ respectively, and then apoptosis rate was analyzed. Data showed A3D8 could significantly enhance DDP-induced apoptosis of spheroid cells (the percentage of apoptotic cells were $5.80 \pm 0.43 \%, 9.93 \pm 1.24 \%, 10.52 \pm 0.51 \%$ and $15.03 \pm 1.23 \%$ for mIgG1, A3D8, DDP and A3D8 plus DDP treatment, respectively) (Figure 4(a)). In further mechanism study, we detected two key indicators in mitochondrial apoptotic pathway (including bcl-2 expression and change of mitochondrial $\Delta \psi M$ ). The results showed that A3D8 could downregulate bcl-2 expression and change mitochondrial $\Delta \psi M$ (Figure 4(b) and (c)). It indicated that A3D8 enhanced DDP-induced apoptosis of spheroid cells, which was relative with mitochondrial apoptotic pathway, to some extent.

\subsection{A3D8 promoted differentiation of spheroid cells}

To investigate whether A3D8 has effects on differentiation of spheroid cells, we treated freshly trypsinized spheroid cells with $\mathrm{mIgG} 1$ or A3D8 at $10 \mu \mathrm{g} / \mathrm{mL}$ for $7 \mathrm{~d}$ respectively. We discovered that, compared with those treated with mIgG1, spheroid cells treated with A3D8 adhered to the plate, acquired an epithelial morphology (Figure 5(a)). We also evaluated expression of epithelial OVCA markers (such as CK-7 and CA125) by immunocytochemistry assay. The data demonstrated that A3D8 significantly promoted expression of CK-7 and CA125, and increased percentage of CK-7 positive cells to $39 \pm 5 \%$, and percentage of CA125 positive cells to $46 \pm 6 \%$ (Figure $5(\mathrm{~b})$ ). The data indicated that A3D8 could promote differentiation of spheroid cells.

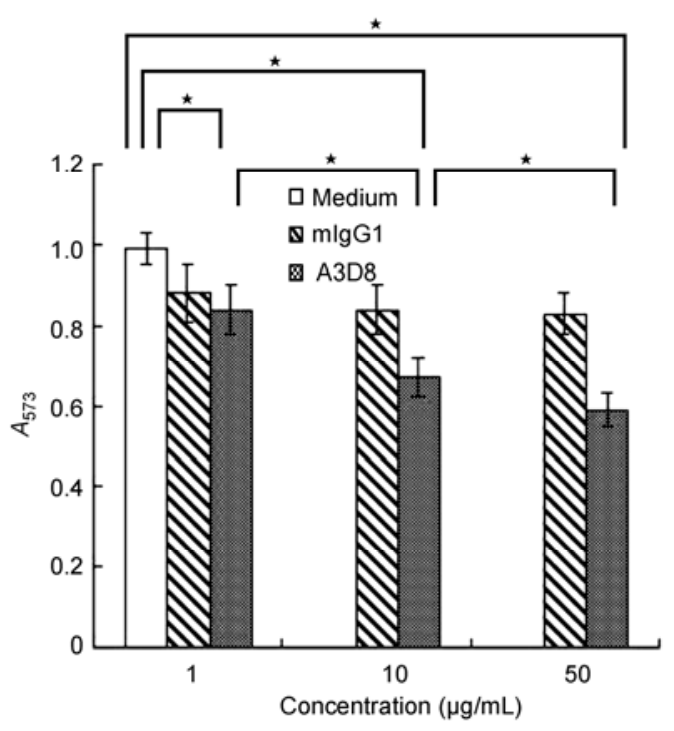

Figure 2 A3D8 inhibited proliferation rate of spheroid cells from SKOV-3. Spheroid cells $\left(1 \times 10^{5}\right.$ cells $\left./ \mathrm{mL}\right)$ were treated with different concentration of $\mathrm{mIgG} 1$ or A3D8 at 1,10 and $50 \mu \mathrm{g} / \mathrm{mL}$ respectively for $48 \mathrm{~h}$. Cell proliferation rate was determined using MTT assay. A3D8 decreased proliferation rate of spheroid cells in a dose-dependent manner. Data are means from three measurements; error bars show standard deviations, $\star$ indicates $P<0.05$. 


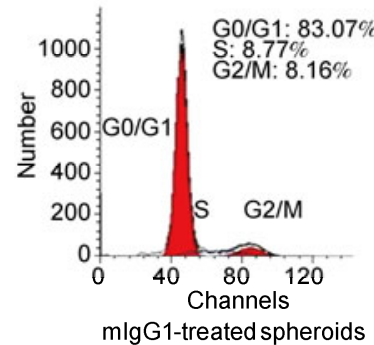

(a)

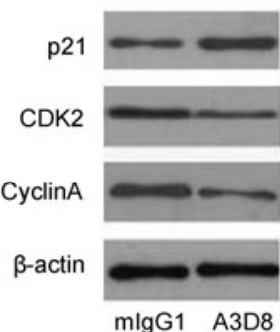

(d)

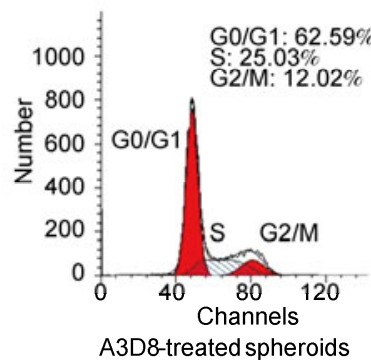

(b)

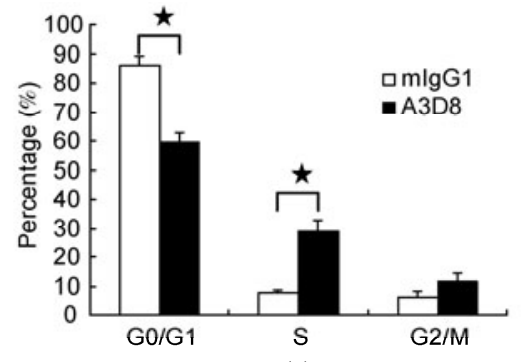

(c)

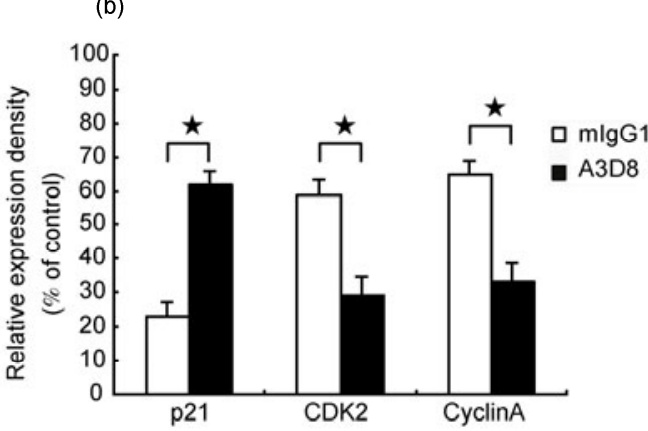

(e)

Figure 3 (Color online) A3D8 changed cell cycle distribution of spheroid cells from SKOV-3. Spheroid cells were pretreated with mIgG1 or A3D8 at 10 $\mu \mathrm{g} / \mathrm{mL}$ for $48 \mathrm{~h}$ respectively, and cell cycle analysis by PI staining using flow cytometry was performed. Plot data showed that A3D8 decreased percentage of G0/G1 while increased percentage of S phase. Histogram data of three independent tests were shown. Cells were pretreated with mIgG1 (a) or A3D8 (b). (c) Bar diagram showed the result of three separate experiments. (d) Western blot results showed that A3D8 could upregulate expression of p21 while downregulate that of CDK2 and cyclinA. (e) Bar diagram showed the result of three separate experiments. Data are means from three measurements; error bars show standard deviations, $\star$ indicates $P<0.05$.

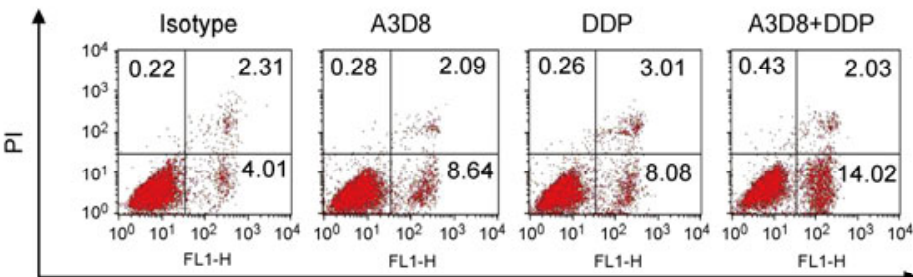

Annexin V-FITC

(a)

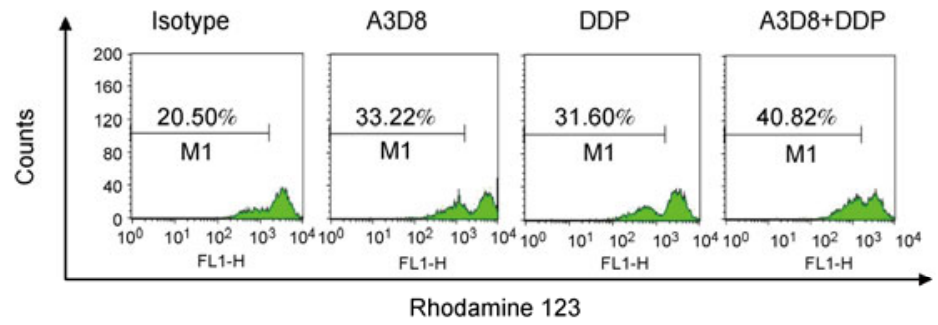

(c)
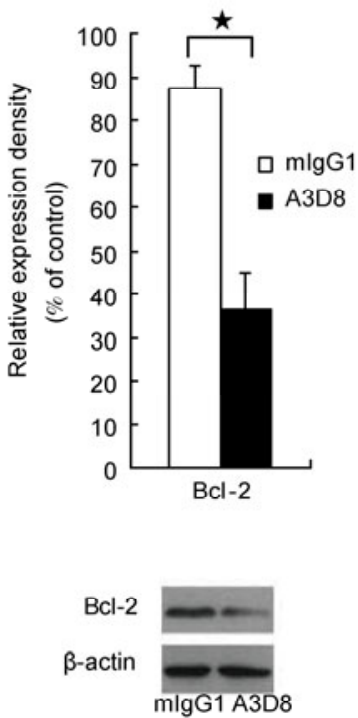

(b)

Figure 4 (Color online) A3D8 enhanced DDP-induced apoptosis of spheroid cells from SKOV-3. (a) Spheroid cells were treated with $10 \mu \mathrm{g} / \mathrm{mL}$ mIgG1 or $10 \mu \mathrm{g} / \mathrm{mL}$ A3D8 or $2.5 \mu \mathrm{g} / \mathrm{mL}$ DDP or $10 \mu \mathrm{g} / \mathrm{mL}$ A3D8 plus $2.5 \mu \mathrm{g} / \mathrm{mL}$ DDP for $24 \mathrm{~h}$ respectively, and flow cytometry analysis results of spheroid cells were shown. (b) Western blot results showed that A3D8 could downregulate bcl-2 expression. Data are means from three measurements; error bars show standard deviations, $\star$ indicates $P<0.05$. (c) Flow cytometry analysis of Rh123 staining showed that A3D8 could change mitochondrial $\Delta \psi M$ of spheroid cells.

\subsection{A3D8 inhibited CFE of spheroid cells}

To determine whether A3D8 has effects on clone-formation of spheroid cells, we pretreated freshly trypsinized spheroid cells with A3D8 at $10 \mu \mathrm{g} / \mathrm{mL}$ for $48 \mathrm{~h}$, and then CFE was assessed. The data demonstrated that CFE of spheroid cells pretreated with A3D8 was obviously lower than that pretreated with $\mathrm{mIgG1}(P<0.05)$ (Figure 6). 


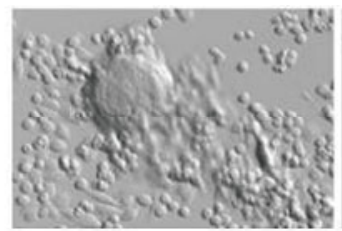

$1 \mathrm{~d}$

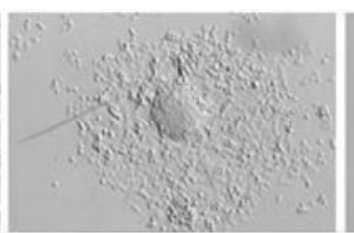

$2 \mathrm{~d}$

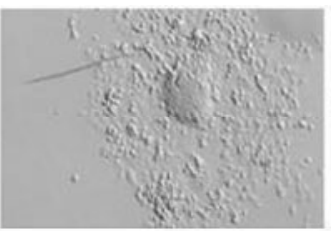

4 d

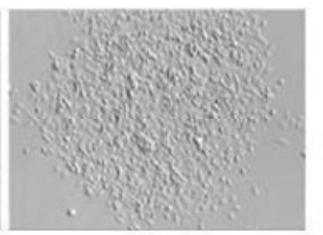

$7 \mathrm{~d}$

(a)
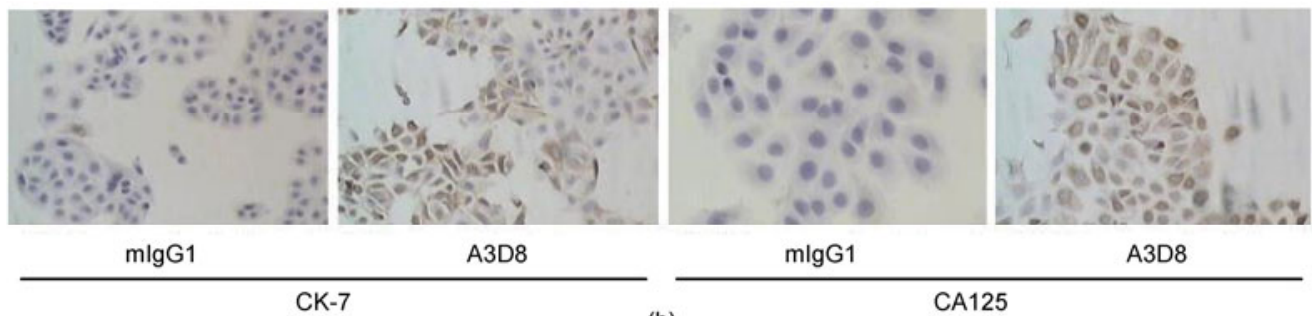

(b)

Figure 5 (Color online) A3D8 promoted differentiation of spheroid cells. The spheroid cells were pretreated with $\mathrm{mIgG} 1 \mathrm{or}$ A3D8 at $10 \mu \mathrm{g} / \mathrm{mL}$ for $48 \mathrm{~h}$ respectively. The morphology of cells was observed under microscope every day. Expression of CK-7 and CA125 was evaluated by immunocytochemistry assay. (a) Typical morphology change of one spheroid treated with A3D8 for $7 \mathrm{~d}$. (b) Expression of CK-7 and CA125 in spheroid cells treated with mIgG1 versus that treated with A3D8.

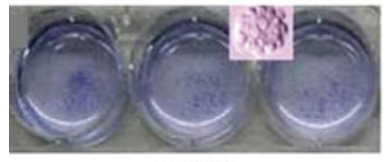

mlgG1

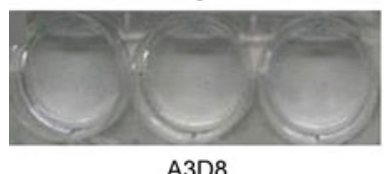

(a)

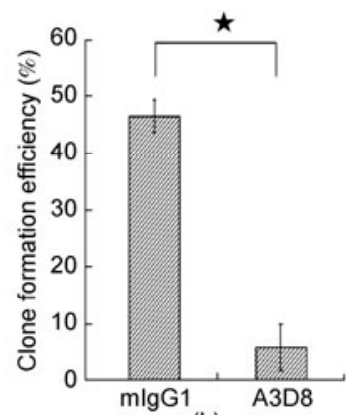

(b)
Figure 6 (Color online) A3D8 inhibited CFE of spheroid cells. (a) The CFE of spheroid cells pretreated with A3D8 was much lower than that of spheroid cells pretreated with the same dose of mIgG1. After 14-d culture, most clones had reached $>50$ cells. (b) The clone that contained more than 50 cells was counted under a microscope. The data showed that A3D8 inhibited significantly CFE of spheroid cells. Data are means from three measurements; error bars show standard deviations, $\star$ indicates $P<0.05$.

\subsection{A3D8 reduced invasive efficacy of spheroid cells}

To investigate whether A3D8 has effects on invasion of spheroid cells, we pretreated freshly trypsinized spheroid cells with A3D8 at $10 \mu \mathrm{g} / \mathrm{mL}$ for $48 \mathrm{~h}$, and then transferred them into transwell-plate pretreated with fibronectin and matrigel. After incubation for $48 \mathrm{~h}$, invaded cells were evaluated. The data showed that, compared with control, A3D8 significantly reduced invasive efficacy of spheroid cells $(P<0.05)$ (Figure 7).

\subsection{A3D8 decreased tumorigenicity of spheroid cells in NOD/SCID mice}

In order to evaluate effect of A3D8 on tumorigenesis in vivo we injected A3D8-pretreated spheroid cells $\left(1 \times 10^{4}\right.$ or $\left.1 \times 10^{5}\right)$ into NOD/SCID mice, with mIgG1-pretreated spheroid cells as control. Sixteen weeks later, all mice were sacrificed and tumors were retrieved for pathologic analysis. The results showed that, in two control groups, 2 of 3 mice $\left(1 \times 10^{4}\right.$ cells $)$, and 3 of 3 mice $\left(1 \times 10^{5}\right.$ cells) respectively reproduced tumor; however, in two tested groups, none of 3 mice $\left(1 \times 10^{4}\right.$ cells) and 1 of 3 mice $\left(1 \times 10^{5}\right.$ cells $)$ respectively reproduced tumor. The results indicated that A3D8 significantly decreased tumorigenesis of spheroid cells in NOD/SCID mice (Table 2).

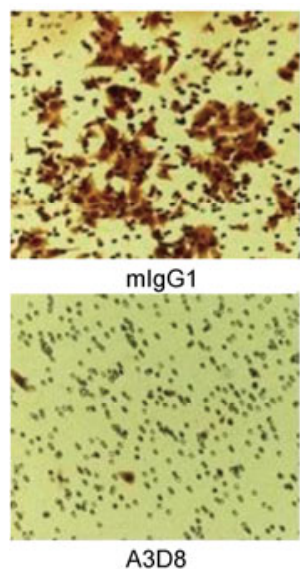

(a)

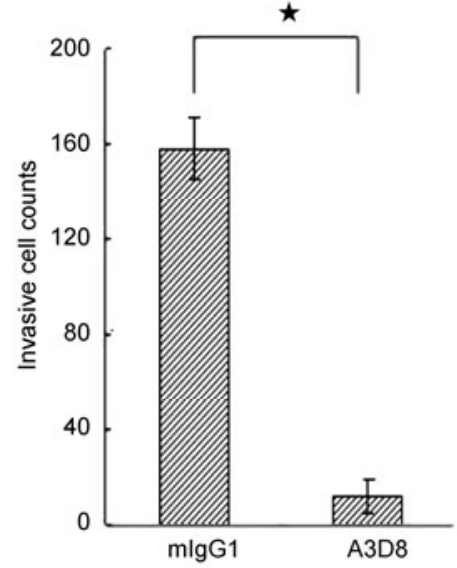

(b)
Figure 7 (Color online) A3D8 inhibited invasive efficacy of spheroid cells from SKOV-3. (a) The spheroid cells were pretreated with mIgG1 or A3D8 at $10 \mu \mathrm{g} / \mathrm{mL}$ for $48 \mathrm{~h}$ respectively, and then transferred them to transwell chamber pretreated with fibronectin and matrigel. After 48-h incubation, membrane of each well was stained with HE. (b) Cells number on lower surface of filter was counted under a microscope. The data showed that A3D8 significantly inhibited invasive efficacy of spheroid cells. Data are means from three measurements; error bars show standard deviations, $\star$ indicates $P<0.05$. 
Table 2 A3D8 inhibited tumorigenicity of spheroid cells in NOD/SCID mice

\begin{tabular}{ccc}
\hline Groups & Cell dose & Tumor formation $^{\text {a) }}$ \\
\hline \multirow{2}{*}{ mIgG1-pretreated spheroid cells } & $1 \times 10^{4}$ & $2 / 3$ \\
& $1 \times 10^{5}$ & $3 / 3$ \\
\hline \multirow{2}{*}{ A3D8-pretreated spheroid cells } & $1 \times 10^{4}$ & $0 / 3$ \\
& $1 \times 10^{5}$ & $1 / 3$ \\
\hline
\end{tabular}

a) Three mice per group.

\section{Discussion}

Mounting evidence demonstrated that CSCs play an important role in tumor progression. Novel strategies targeting CSCs have been widely explored. The present study demonstrated that there existed a small population with so-called "stemness" property in well-established human OVCA cell line (SKOV-3) and anti-CD44 mAb could remodel some biological behavior of such subpopulation.

Recently, it is assumed that many established malignant cell lines contain a rare subpopulation of stem cells that can be maintained indefinitely in culture, and play crucial role in malignancy [22-26]. Moreover, some studies reported that minor subpopulation is enriched in side population (SP), and there is a positive correlation between SP cells and stem cells [24,27]. Therefore, first of all we detected SP cells in four well-establish human OVCA cell lines (including SKOV-3, OVCAR-3, Caov-3, and HO-8910). The data illustrated percentage of SP cells in above four cell lines is about $0.6 \pm 0.1 \%, 0.1 \pm 0.1 \%, 0.3 \pm 0.2 \%, 0.2 \pm 0.2 \%$ respectively. Among four cell lines, percentage of SP cells in SKOV-3 is the highest (Figure 8). Therefore we next managed to isolate stem/progenitor cells from SKOV-3.

To date, in general, there are several methods to isolate tumor cells with stem/progenitor property, including (1) isolating SP by flow cytometry as CSCs [24,27]; (2) isolating so-called CSCs by specific surface markers [28,29]; (3) isolating spheroid cells in serum-free culture condition $[13,14,30,31]$. So in the present study, we exploited method described by Bapat et al. [14] to isolate spheroid cells from SKOV-3 cells in serum-free culture condition. As to the identification of CSCs, putative criteria includes following aspects: firstly, like normal adult stem cells, CSCs exhibit the ability to unlimitedly divide as well as give rise to differentiated tissue cells in vitro [13,14,19,32]; secondly, CSCs express putative stem cell-relevant markers (such as Nanog, Oct4, ABCG2, etc.) [14,20]; thirdly, quiescence of CSCs, namely most CSCs are in G0/G1 phase in cell cycle [33]; fourthly, a small quantity of so-called CSCs can reproduce tumor in NOD/SCID mice [13,14,19,32]. Accordingly, we identified isolated cells from several aspects described above. Our data showed that spheroid cells grew exponentially in vitro in serum-free medium in an anchorindependent manner for more than 6 months without obvious phenotype change; cell cycle analysis showed that about
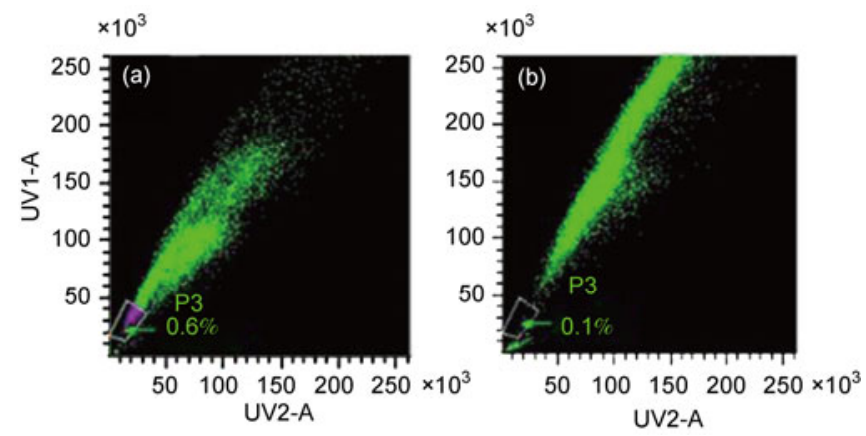

Figure 8 (Color online) SKOV-3 cell line contained a small quantity of SP (side population). Cells from SKOV-3 were stained with Hoechst 33342 and analyzed by flow cytometry. (a) The SP cells were outlined and shown as a percentage of total cell population. (b) This cell population disappeared in the presence of verapamil.

$85.23 \pm 2.36 \%$ cells were in G0/G1 phase; after adding $10 \%$ serum to conditioned medium, spheroid cells expressed putative epithelial OVCA differentiation antigen, such as CK-7 and CA125; these spheroid cells expressed putative stem/progenitor cell-relevant marker proteins, such as Oct-4, Nanog, and ABCG2; and these spheroid cells also had high ALDH activity. Moreover, only $1 \times 10^{4}$ spheroid cells injected into NOD/SCID mice had the ability to reproduce tumor with similar phenotype to that reproduced by $1 \times 10^{6}$ adherent SKOV-3 cells. Our data implied existence of a small subpopulation that had so-called "stemness" in SKOV-3 cell line and could be propagated.

Thereafter, we evaluated influence of anti-CD44 mAb (A3D8) on behaviors of the above spheroid cells with stem/progenitor property. First, we detected expression of CD44 molecule on the surface of spheroid cells. The plot data showed that about $88 \%$ spheroid cells expressed CD44 at high level (Figure 9). That is not only similar to the results reported by Zhang et al. [13] but also is the basis of our following experiments.

CD44 comprises a family of membrane adhesion molecules encoded by a single gene and diversified by alternative splicing and extensive posttranslational modifications, known as CD44 variant isoforms (generally including CD44s and CD44v). The expression pattern of CD44s and relation with biological properties of tumors are dependent
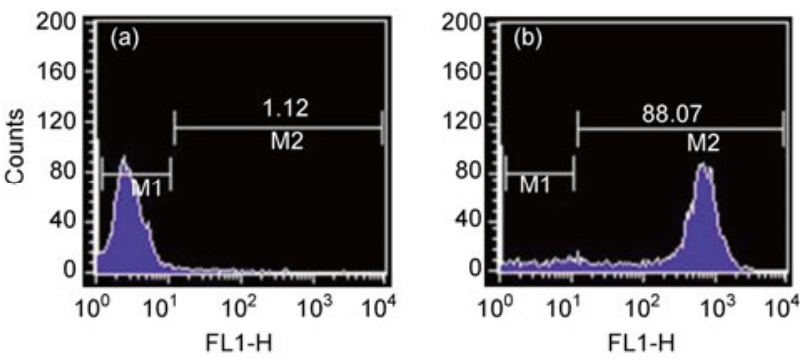

Figure 9 (Color online) Expression of CD44 in spheroid cells: (a) isotype control; (b) test group. Three independent experiments were done, and percentage of CD44 positive cells was $86.34 \pm 1.58 \%$. 
upon tumor type and origin [34]. Cho et al. [35] demonstrated that overexpression of CD44s was significantly associated with high tumor grade of ovarian neoplasms.

A3D8, purified from A3D8 hybridoma, is a kind of mouse monoclonal anti-CD44 antibodies and may recognize all CD44 isoforms [15]. Since CD44 is strongly expressed on surface of many human cancer cells and CSCs, anti-CD44 antibodies are widely-used in anti-cancer studies [8-11]. Recently, it is reported that targeting CD44 with activating mAbs has led to eradication of human LSCs [12]. This indicates that such CSC-targeting therapy may be a promising approach for cancer treatment. However, there are few reports about antibody-based CSC-targeting therapy for solid tumor, such as OVCA. Accordingly, we used A3D8 to treat spheroid cells from SKOV-3, one of OVCA cell lines, and conducted a batch of testing to evaluate influence of $\mathrm{A} 3 \mathrm{D} 8$ on behaviors of spheroid cells with "stemness". Our data showed that A3D8 could significantly inhibit proliferation of spheroid cells and promote them to differentiate. These results are similar to results reported by Gadhoum et al. [11].

Apoptosis detection test in our present study showed that apoptosis of spheroid cells was significantly enhanced after DDP treatment combined with A3D8. The analysis of mitochondrial transmembrane potential and bcl-2 expression supported the data from apoptosis assay. We proposed that A3D8 may downregulate bcl-2 expression and then decrease stability of mitochondrial membrane and cause eventual cell death. The results supported that A3D8 had advantage on increasing chemosensitivity of spheroid cells to conventional chemotherapy drugs (such as DDP).

Cell cycle analysis in our current study showed that A3D8 could reduce fraction in G0/G1 phase, companied by $\mathrm{S}$-phase arrest, of spheroid cells. It has been reported that high portion of G1 in tumor cells was closely related to chemo-resistant and anti-apoptotic behaviors [33,36]. Thus we supposed that cell cycle distribution change of spheroid cells described above may be a presumable reason for decreased proliferation by A3D8 and also for enhanced apoptosis of spheroid cells induced by A3D8 plus DDP compared to DDP alone. The data also showed that A3D8 could regulate expression of $\mathrm{p} 21, \mathrm{CDK} 2$ and cyclinA, which are important protein regulators in cell cycle. These results indicated that there might be a correlation existing between decrease in the fraction of spheroid cells in G0/G1 phase with $S$ arrest and changes of p21, CDK2 or cyclinA expression.

It is notable that OVCA may spread by implantation of tumor cells onto mesothelial lining of peritoneal cavity [37]. One important mechanism is interaction between hyaluronic acid and CD44 receptor [38]. A conventional curative treatment is however difficult to achieve. Therefore, preventing tumor-cells binding to mesothelium is crucial for patients' outcome. It is possible that strategies to interfere CD44s function may decrease chance of intra-abdominal spread of this highly lethal neoplasm [37]. Thus, in the cur- rent study, we employed in vitro model of invasion assay using matrigel-coated membrane to measure the influence of A3D8 on invasion ability of spheroid cells. Our data demonstrated that A3D8-pretreated spheroid cells had very low invasion potential. The result indicated that A3D8 might interfere with spreading process of OVCA cells. Additionally, our data showed that A3D8 strongly decreased CFE of spheroid cells. This result indicated that A3D8 might also decrease possibility of tumor-reproduction in a relocated site. Results also showed that A3D8 could decrease tumorigenicity of spheroid cells in NOD/SCID mice. All these data ensure potential of A3D8 to be a novel and promising tool in target-strategy against OVCA.

In conclusion, for the first time, we isolated and identified spheroid cells with so-called "stemness" property from human OVCA cell line SKOV-3; and our present study revealed that anti-CD44 therapy may become a novel and promising target-strategy for OVCA treatment.

This work was supported by the National Natural Science Foundation of China (30670801) and the Tianjin Research Program of Application Foundation and Advanced Technology (O6YFJMJC08300).

1 Mimeault M, Batra S K. Functions of tumorigenic and migrating cancer progenitor cells in cancer progression and metastasis and their therapeutic implications. Cancer Metast Rev, 2007, 26: 203-214

2 Dean M, Fojo T, Bates S. Tumor stem cells and drug resistance. Nat Rev Cancer, 2005, 5: 275-284

3 Mimeault M, Hauke R, Mehta P P, et al. Recent advances in cancer stem/progenitor cell research: Therapeutic implications for overcoming resistance to the most aggressive cancers. J Cell Mol Med, 2007, 11: $981-1011$

4 Brabletz T, Jung A, Spaderna S, et al. Opinion: Migrating cancer stem cells-An integrated concept of malignant tumor progression. Nat Rev Cancer, 2005, 5: 744-749

5 Miller S J, Lavker R M, Sun T T. Interpreting epithelial cancer biology in the context of stem cells: Tumor properties and therapeutic implications. Biochim Biophys Acta, 2005, 1756: 25-52

6 Massard C, Deutsch E, Soria J C. Tumor stem cell-targeted treatment: Elimination or differentiation. Ann Oncol, 2006, 17: 1620-1624

7 Ponnusamy M P, Batra S K. Ovarian cancer: Emerging concept on cancer stem cells. J Ovarian Res, 2008, 1: 4-12

8 Misaghian N, Ligresti G, Steelman L S, et al. Targeting the leukemic stem cell: The Holy Grail of leukemia therapy. Leukemia, 2008, 23: 25-42

9 Charrad R S, Gadhoum Z, Qi J Y, et al. Effects of anti-CD44 monoclonal antibodies on differentiation and apoptosis of human myeloid leukemia cell lines. Blood, 2002, 99: 290-299

10 Gadhoum Z, Delaunay J, Maquarre E, et al. The effect of anti-CD44 monoclonal antibodies on differentiation and proliferation of human acute myeloid leukemia cells. Leuk Lymphoma, 2004, 45: 1501-1510

11 Gadhoum Z, Leibovitch M P, Qi J Y, et al. CD44: A new means to inhibit acute myeloid leukemia cell proliferation via p27 Kip1. Blood, 2004, 103: 1059-1068

12 Jin L Q, Hope K J, Zhai Q L, et al. Targeting of CD44 eradicates human acute myeloid leukemic stem cells. Nat Med, 2006, 12: 1167-1174

13 Zhang S, Balch $\mathrm{C}$, Chan M W, et al. Identification and characterization of ovarian cancer-initiating cells from primary human tumors. Cancer Res, 2008, 68: 4311-4320

14 Bapat S A, Mali A M, Koppikar C B, et al. Stem and progenitor-like cells contribute to the aggressive behavior of human epithelial ovari- 
an cancer. Cancer Res, 2005, 65: 3025-3029

15 Patel D D, Hale L P, Whichard L P, et al. Expression of CD44 molecules and CD44 ligands during human thymic fetal development: Expression of CD44 isoforms is developmentally regulated. Int Immunol, 1995, 7: 277-286

16 Li Z, Shen X T, Cao L, et al. Bone morphogenetic protein 2 improves patellar tendon healing by promoting migration and proliferation of tenocytes. Chin Sci Bull, 2011, 56: 1361-1369

17 Li H Z, Wang Y, Gao Y, et al. Effects of raf kinase inhibitor protein expression on metastasis and progression of human epithelial ovarian cancer. Mol Cancer Res, 2008, 6: 917-928

18 Matsui W, Wang Q, Barber J P, et al. Clonogenic multiple myeloma progenitors, stem cell properties, and drug resistance. Cancer Res, 2008, 68: 190-197

19 Takubo K, Ohmura M, Azuma M, et al. Stem cell defects in ATMdeficient undifferentiated spermatogonia through DNA damageinduced cell-cycle arrest. Cell Stem Cell, 2008, 2: 170-182

20 Pascal L E, Oudes A J, Petersen T W, et al. Molecular and cellular characterization of ABCG2 in the prostate. BMC Urol, 2007, 7: 6-18

21 Croker A K, Goodale D, Chu J, et al. High aldehyde dehydrogenase and expression of cancer stem cell markers selects for breast cancer cells with enhanced malignant and metastatic ability. J Cell Mol Med, 2009, 13: 2236-2252

22 Wang J, Guo L P, Chen L Z. Identification of cancer stem cell-like side population cells in human nasopharyngeal carcinoma cell line. Cancer Res, 2007, 67: 3716-3724

23 Kondo T, Setoguchi T, Taga T. Persistence of a small subpopulation of cancer stem-like cells in the C6 glioma cell line. Proc Natl Acad Sci USA, 2004, 101: 781-786

24 Maria M H, Alvin V N, Stephen L, et al. Side population in human lung cancer cell lines and tumors is enriched with stem-like cancer cells. Cancer Res, 2007, 67: 4827-4833

25 Li Y F, Xiao B, Lai Z S, et al. Spheres isolated from Colo205 cell line possess cancer stem-like cells under serum-free culture condition. J South Med Univ, 2008, 28: 236-240
26 Dou J, Pan M, Wen P, et al. Isolation and identification of cancer stem-like cells from murine melanoma cell lines. Cell Mol Immunol, 2007, 4: 467-472

27 Wu C, Wei Q, Utomo V, et al. Side population cells isolated from mesenchymal neoplasms have tumor initiating potential. Cancer Res, 2007, 67: 8216-8222

28 Al-Hajj M, Wicha M S, Benito-Hernandez A. Prospective identification of tumorigenic breast cancer cells. Proc Natl Acad Sci USA, 2003, 100: 3983-3988

29 Singh S K, Hawkins C, Clarke I D, et al. Identification of human brain tumour initiating cells. Nature, 2004, 432: 396-401

30 Hemmati H D, Nakano I, Lazareff J A, et al. Cancerous stem cells can arise from pediatric brain tumors. Proc Natl Acad Sci USA, 2003, 100: $15178-15183$

31 Singh S K, Clarke I D, Terasaki M, et al. Identification of a cancer stem cell in human brain tumors. Cancer Res, 2003, 63: 5821-5828

32 Tan B T, Park C Y, Ailles L E, et al. The cancer stem cell hypothesis: A work in progress. Lab Invest, 2006, 86: 1203-1207

33 Gao G, Dou Q P. G(1) phase-dependent expression of bcl-2 mRNA and protein correlates with chemoresistance of human cancer cells. Mol Pharmacol, 2000, 58: 1001-1010

34 Makrydimas G, Zagorianakou N, Zagorianakou P, et al. CD44 family and gynaecological cancer. In Vivo, 2003, 17: 633-640

35 Cho E Y, Choi Y, Chae S W, et al. Immunohistochemical study of the expression of adhesion molecules in ovarian serous neoplasms. Pathol Int, 2006, 56: 62-70

36 Knowlton K, Mancini M, Creason S, et al. Bcl-2 slows in vitro breast cancer growth despite its antiapoptotic effect. J Surg Res, 1998, 76: 22-26

37 Cannistra S A, Kansas G S, Niloff J, et al. Binding of ovarian cancer cells to peritoneal mesothelium in vitro is partly mediated by $\mathrm{CD} 44 \mathrm{H}$. Cancer Res, 1993, 53: 3830-3838

38 Zada A A, Singh S M, Reddy V A, et al. Downregulation of c-Jun expression and cell cycle regulatory molecules in acute myeloid leukemia cells upon CD44 ligation. Oncogene, 2003, 22: 2296-2308

Open Access This article is distributed under the terms of the Creative Commons Attribution License which permits any use, distribution, and reproduction in any medium, provided the original author(s) and source are credited. 